\title{
EL VALOR ECONÓMICO AGREGADO (EVA) EN EL VALOR DEL NEGOCIO
}

\author{
FEDERICO LI BONILLA \\ Escuela de Ciencias de la Administración \\ Universidad Estatal a Distancia, Costa Rica
}

fli@uned.ac.cr

\section{RESUMEN}

El Valor Económico Agregado (EVA) es el importe que queda en una empresa una vez cubiertas la totalidad de los gastos y la rentabilidad mínima proyectada o estimada por los administradores. La principal innovación del EVA es, por un lado, la incorporación del costo del capital en el cálculo del resultado del Negocio y, por otro, la modificación del comportamiento de los administradores, quienes pasan a actuar como si fueran ellos mismos los accionistas, y esperaran, al igual que éstos, el mayor rédito por sus inversiones en la empresa. El EVA es una herramienta que brinda información imprescindible sobre ciertos indicadores financieros a la hora de analizar los resultados de la gestión financiera; entre ellos, los factores que inciden en la generación de valor en la empresa, y específicamente, en el Valor del Negocio. Adicionalmente, el concepto incorpora activos que casi nunca se toman en cuenta y no aparecen en los estados financieros de las empresas como activos intangibles; por ejemplo, el Valor del Conocimiento, el cual se encuentra depositado en los colaboradores de la organización.

PALABRAS CLAVE: COSTO DEL CAPITAL, INNOVACIÓN, INTANGIBLES, VALOR DEL NEGOCIO, VALOR DEL CONOCIMIENTO.

\section{ABSTRACT}

Nowadays, there is a growing tendency to give proper recognition to some intangible factors that increase the value of a business, basically by means of a technique called EVA (economic value added). This technique takes into account the increased productivity and motivation of human resources, the incorporation of information and communication technology (IT) in the firm, as well as the value of the brand. All together, they raise the value of a firm above the simple accounting value reflected in traditional accounting techniques and methods.

KEYWOKS: COST OF CAPITAL, INNOVATION, INTANGIBLE ASSETS, BUSINESS VALUE, VALUE OF KNOWLEDGE.

\section{INTRODUCCIÓN}

Los grandes cambios en la manera de hacer negocios hoy en día, debidos en gran parte al hecho de contar con una clientela más informada y muy exigente, hacen que las empresas de los numerosos sectores en el ámbito mundial no solo se vean empujadas a cambiar, sino que también deban ajustarse a factores como la liberalización y la globalización de los negocios, así como las implicaciones que ambos procesos tienen en las tecnologías y que, entre otras cosas, las llevan a una constante innovación tecnológica.

La necesidad de ser eficientes y eficaces en estos tiempos, para poder ser siempre atractivos a los inversores y generar en ellos la confianza necesaria para que inviertan sus recursos en una actividad que genere dividendos competitivos en relación con los diferentes sectores de hacer negocio, ha 
obligado a los administradores a implantar nuevos parámetros para medir el Valor del Negocio.

El inicio del siglo XXI ha estado marcado por la necesidad de rediseñar la manera de valuar las diferentes formas y métodos para generar más valor al negocio, sea éste de agroindustria, minería, textiles, telecomunicaciones, banca, seguros, electrónica, industria automotriz, aeronáutica, diseños de procesadores para computadoras, software, medicina, servicios públicos (estatales), o servicio de información, entre otros.

Para generar mayor valor al negocio, se recurre a diferentes técnicas métricas que permiten cuantificarlo, entre ellas, la creación de Valor para el accionista, así como el Valor del conocimiento, Valor del cliente, Valor del proveedor, Valor del recurso humano, Valor de las Tecnologías, Información y Comunicación (TIC), Valor al medio ambiente; Valor a la comunidad. Adicionalmente, se presta una mayor importancia a los activos intangibles, los cuales generan un mayor Valor del Negocio; de ahí la importancia de otorgar especial atención al Valor de la marca, las patentes y los derechos de autor, entre otros.

En el Valor del Negocio se pone especial atención a los activos tangibles, los cuales no son generadores bondadosos en la creación del valor, sino que, más bien, en muchas ocasiones lo destruyen; por eso se mantienen los activos en un nivel mínimo o apenas suficiente para cubrir solamente una instalación de producción, o bien, se opta por operar con el mínimo de activos, de manera que los activos destructores de valor tiendan a cero.

Como se indicó anteriormente, en el actual contexto de globalización de las empresas, se tiene una exigente clientela cada vez más informada, educada y con un mayor acceso del conocimiento en el entorno. Esto ha hecho que para dar mayor Valor al Negocio, se recurra a aspectos como la innovación, la valorización de los empleados (generadores de riquezas y reservorios del conocimiento), la simplificación de las informaciones dentro y fuera de la empresa, y el desarrollo de encadenamientos fuertes, intensos y multiplicativos, que permitan mantener ventajas competitivas. El objetivo concreto de contar con los aspectos indicados, es que la empresa pue$\mathrm{da}$, ante todo, ajustarse a las exigencias de estos tiempos, pero hacerlo creando valor.

Hoy, empresas como IBM, Kodak, Motorola, Xerox, Hewlett-Packard, GMC, Ford, Coca Cola, Microsoft, Intel, Telecom., JC Penny, AT\&T, Quaker, Durman Esquivel; entre más de trescientas empresas de Europa, Asia, Norteamérica y Latinoamérica, echan mano de las diferentes métricas para medir la creación o destrucción del valor; sin embargo, de todas esas métricas, la de mayor uso es el EVA: "Valor Económico Agregado".

Esta investigación pretende contribuir al uso de modelos para cuantificar la creación o destrucción del valor, en las diferentes organizaciones.

\section{MARCO TEÓRICO}

El concepto del EVA es una variación o modificación de lo que tradicionalmente se conocía como "Ingreso o beneficio residual", es decir, el resultado que se obtenía al restar a la utilidad operacional los costos del capital. La idea del beneficio residual apareció a principios del siglo XX, en la literatura contable. Church lo introdujo en 1917 y luego, en 1924, Scovell citó el beneficio residual; ya para 1960 aparece dicho concepto y se contempla en la literatura de la contabilidad gerencial. Diez años más tarde, para la década de los 70, los académicos finlandeses discuten sobre dicho tema y en 1975 Virtanen lo define como un retorno sobre inversión, para la toma de decisiones gerenciales.

En 1989, la firma consultora Stern Stewart \& Co., radicada en Nueva York, Estados Unidos de América, reintrodujo y registró como marca a su nombre el EVA. 
Para crear y cuantificar el Valor del Negocio hay varias métricas, pero, como se indicó antes, las más utilizadas son: el "EVA" o Valor Económico Agregado, el "VMA" o Valor de Mercado Agregado, el flujo de caja descontado, entre otros.

En el análisis de los activos, los activos intangibles son los que más aportan a la creación de ganancias en el Valor del Negocio, entre ellos sobresalen las patentes, los derechos de autor, las marcas, el capital intelectual (Gestión del Conocimiento), las Tecnologías de Información y Comunicación (TICS).

\section{¿Cómo se crea Valor en el Negocio?}

Se podría responder a dicha interrogante diciendo que se crea valor cuando los administradores (de la empresa o del negocio), hacen las cosas bien; es decir, cuando las acciones tomadas generan una rentabilidad económica superior al costo de los recursos económicos o capital utilizados en el giro empresarial. Los resultados de esta aseveración se detallan a continuación (Parro, 1972; Viñolas \& Adserà, 1997; Amat, 1999; HBR, 1999; Young, 2001; Stern, 2002; Irimia, 2003; Martín \& de la Calle, 2003; Sanjurjo \& Reinoso, 2003; Rappaport, 2006):

1. La rentabilidad económica no es igual a la rentabilidad contable. La rentabilidad económica es la generación de flujos de fondos, los cuales no pueden ser maquillados por la contabilidad creativa; en tanto la rentabilidad contable es la relación de datos contables (relación de beneficio contable / recurso contable), esto se visualiza en el verdadero rendimiento económico (creando valores en el negocio: dinero), en tanto el beneficio contable es histórico y normalmente queda registrado en libros.

2. Los recursos utilizados para obtener estos flujos, tienen un costo económico.
3. La creación de valor en el negocio se refiere a expectativas futuras, no a referencias pasadas o históricas.

4. En relación con las expectativas, éstas deben estar bien planificadas y fundamentadas para ser realistas y alcanzables, lo que supone un compromiso serio y profundo para poder obtener las metas fijadas; estableciendo mecanismos de seguimiento para cuantificar el logro y corregir las desviaciones.

5. Al crear valor se hace referencia a un futuro, el cual siempre es incierto, por lo que la rentabilidad económica esperada está asociada a un nivel de riesgo que, en la medida de lo posible, hay que controlar, conocer y asumir.

6. Crear valor es parte fundamental de la visión y la misión de toda la organización empresarial y no de unos cuantos colaboradores. Como corolario a la creación de Valor en el Negocio, se debe tener un cambio total y profundo, de mejoramiento continuo, de educación y compromiso que abarque todos los niveles: desde la Junta Directiva, Directores, Gerentes hasta el Personal de toda la organización.

En la creación del Valor del Negocio, se necesita la voluntad de los altos directivos, el seguimiento de los mandos intermedios y la voluntad de los demás colaboradores para con la empresa; su implantación, además, debe ser convincente, profunda, estrecha y realizada con tiempo, a fin de obtener rendimientos económicos a futuro (largo plazo). La confianza es algo intrínseco al Valor del Negocio, y para lograrlo es necesario contar con firmes encadenamientos a largo plazo con proveedores, clientes, colaboradores, entorno social, entorno ecológico, accionistas y acreedores, entre otros. No se debe olvidar el mejoramiento continuo y la calidad total, el evitar gastos innecesarios en activos tangibles, y cada vez apostar más por el conocimiento y las nuevas Tecnologías de Información y Comunicación. (TICS) 


\section{La Medición de la creación del Valor}

Para saber si realmente se ha creado valor, se debe medir dicha creación económica en el negocio o empresa. Para ello, es necesario conocer los flujos de fondos (dinero) y cuantificar el empleo de los recursos utilizados para la obtención de dichos flujos monetarios; además, se debe medir el riesgo asociado a esas decisiones futuras, para poder así cuantificar la rentabilidad económica que se crea.
Ninguna de las mediciones del desempeño económico es nueva y en la actualidad es posible encontrar y aplicar diferentes cálculos o métricas para cuantificar la creación o destrucción de Valor al Negocio.

En la tabla 1 se presenta algunas de las métricas utilizadas para tal fin:

TABLA 1

\section{DEFINICIÓN DE LAS MÉTRICAS}

\begin{tabular}{|c|c|}
\hline MÉTRICAS & DEFINICIÓN \\
\hline \multicolumn{2}{|l|}{ Métricas contables } \\
\hline Resultado Neto & Resultados después de impuestos \\
\hline Beneficio por acción & Resultado neto entre número de acciones \\
\hline NOPAT & $\begin{array}{l}\text { Resultado antes de intereses e impuestos - impuestos a pa- } \\
\text { gar por operaciones. }\end{array}$ \\
\hline ROE & NOPAT (año t) entre total de Recursos Propios (año t-1) \\
\hline ROA & $\begin{array}{l}\text { NOPAT (año t) dividido por diferencia entre Activos Ope- } \\
\text { rat. y Pasivos sin costo financiero (año t-) } 1\end{array}$ \\
\hline \multicolumn{2}{|l|}{ Métricas basadas en el Flujo de caja (cash flow) } \\
\hline Cash Flow Operativo (CFO) & NOPAT más gastos de amortización \\
\hline Cash Flow libre Operaciones. & $\begin{array}{l}\text { CFO - necesidades operativas de fondos y necesidades } \\
\text { Netas de inversión de Activos Fijos }\end{array}$ \\
\hline Cash Flow Return (CFR) & $\begin{array}{l}\text { CFO entre total de Activos Netos - Pasivo sin costo finan- } \\
\text { ciero }\end{array}$ \\
\hline Return On Gross Investment (ROGI) & $\begin{array}{l}\text { CFO entre total de Activos Bruto - pasivo sin costo finan- } \\
\text { ciero }\end{array}$ \\
\hline \multicolumn{2}{|l|}{ Métricas basadas en la cotización } \\
\hline Valor de la cotización & Valor de mercado de la acción \\
\hline Total Share Return & $\begin{array}{l}\text { Variación de la cotización entre dos años (t y t-1) más dividen- } \\
\text { dos pagado en el año t, dividido por cotización del año t-1 }\end{array}$ \\
\hline \multicolumn{2}{|l|}{ Métricas basadas en el beneficio económico } \\
\hline Beneficio Económico & NOPAT (año t) - Costo de capital (año t) x activos Netos ${ }^{1}$ \\
\hline Cash Value Added (CVA) & $\begin{array}{l}\text { CFOt - Amort. Económica - Kt x (Activos Brutos t-1) } \\
\text { a) Identico al Beneficio Económico }\end{array}$ \\
\hline EVA & $\begin{array}{l}\text { Puede diferir en los ajustes necesarios para llegar a la NOPAT } \\
\text { b)UAIDI }\end{array}$ \\
\hline
\end{tabular}

FuENTE: Elaboración propia basado en Parró (1972), Viñolas y Adserà (1997), Amat (1999), HBR (1999), Young (2001), Stern (2002), Irimia (2003), Martín y de la Calle (2003), Sanjurjo y Reinoso (2003), Rappaport (2006).

1. $\mathrm{Al}$ ser ROAt $=$ NOPATt $/(\mathrm{año} \mathrm{t}-1)$, el beneficio económico se puede expresar como: $(\mathrm{ROAt}-\mathrm{Kt}) \mathrm{x}$ año $-\mathrm{t}$. 


\section{El EVA en el Valor del Negocio}

El valor económico agregado EVA (en inglés, Economic Value Added) es un método de desempeño financiero para calcular el verdadero beneficio económico de una empresa. El EVA puede calcularse restando de la Utilidad Operativa Neta después de impuestos, la carga del costo de oportunidad. Según Amat (1999), "el costo de oportunidad es una forma de valorar el costo que tiene para la empresa el hecho de que se financie con fondos aportados por los accionistas. Este costo está relacionado, esencialmente, con los dividendos" (p. 87).) del capital invertido, que es "el importe que queda una vez que se han deducido de los ingresos, la totalidad de los gastos, incluidos el costo de oportunidad del capital y los impuestos"(Amat, 1999, p. 92).

El EVA (marca registrada de Stern Stewart \& Co.) es una estimación del monto de las ganancias que difieren de la tasa de rentabilidad mínima requerida (contra inversiones de riesgo parecido) para los accionistas o acreedores; siendo la diferencia entre la creación de valor o la destrucción de valor.

\section{Objetivos generales del EVA}

1. El objetivo financiero primario de cualquier negocio, es el maximizar las riquezas de sus accionistas. Según Rappaport (2006): "La teoría de la economía de mercados se basa en que los individuos buscan su propio interés a través de las transacciones de mercado para producir finalmente una asignación eficiente de los recursos." (p.28).

2. El valor de una empresa depende del grado en el cual los inversionistas confíen (crean) que los beneficios futuros difieren del costo de capital. "Entre tanto, la puesta en práctica del valor para el accionista ha contribuido a transformar a la industria americana convirtiéndola en la más competitiva del mundo [...]", (Rappaport, 2006, p. 28).

\section{Objetivos específicos del EVA}

El EVA trata de solucionar las limitaciones y cubrir vacíos que dejan los indicadores tradicionales (Amat, 1999; Young, 2001; Stern, 2002):

1. Implantar en cualquier tipo de empresa (pequeña o transnacional) cotícese o no, en la bolsa de valores.

2. Aplicar tanto a una corporación, como a una parte de ella (centro de responsabilidad, unidades de negocio, filiales).

3. Fijar metas en la organización.

4. Medir del desempeño de la empresa y sus colaboradores. "La innovación y los productos y servicios a la medida del cliente, desarrollados por empleados con elevada preparación profesional, constituyen una fuente crítica de creación de valor que cada vez cobra más importancia" (Rappaport, 2006).

5. Mejorar la comunicación entre los inversionistas y accionistas. El modelo de las partes involucradas*: en el original, stakeholders, es decir, partes interesadas en las actividades, operacionales y resultados de la empresa. * Nota del traductor a partir del texto original de (Rappaport, 2006).

6. Considerar todos los costos que se producen en la empresa. "Por ejemplo, proporcionar un producto comparable con los de la competencia, pero a menor coste, o proporcionar más valor al cliente gracias a una calidad superior, unas características especiales, o unos servicios posventa [...]"(Rappaport, 2006).

7. Motivar a distintos niveles gerenciales."Hay al menos cuatro factores que impulsarán a los directivos a adoptar una orientación en pro de los accionistas: 1) una posición relativamente sustancial en la propiedad de la empresa, 2) una remuneración vinculada al comportamiento del rendimiento del accio- 
nista, 3) la amenaza de absorción por parte de otra empresa, grupo u organización, y 4) mercados de trabajo competitivos para los directores de grandes empresas." Rappaport, (2006).

8. Valuar la empresa."El movimiento hacia las absorciones que tuvo lugar en la segunda mitad de la década de los ochenta fue un poderoso incentivo para que los directivos concentraran su atención en la creación de valor (Rappaport, 2006).

9. Considerar el riesgo con el que opera la empresa.

En los objetivos del EVA, racionalizan las inversiones a corto y largo plazo, con el fin de generar también valor económico en el Valor del Negocio; tratando de medirlo de una forma tan eficaz que puede llevar a realizar hasta 166 ajustes diferentes.

En el Valor del Negocio utilizando el EVA se pone énfasis en los activos intangibles para crear riquezas y evitar la destrucción de valor económico (dinero).

\section{Cálculo y concepto del EVA}

El EVA considera la productividad de todos los factores utilizados para realizar la actividad empresarial. Se crea valor en una empresa cuando la rentabilidad generada supera al costo de oportunidad, con los recursos utilizados por la empresa, con relación al valor que se generaría en una actividad parecida en el entorno.

En otras palabras, el Valor Económico Agregado $(E V A)^{2}$ es la utilidad operacional después de impuestos (NOPAT) menos costo promedio de capital. En relación con este índice, si la rentabilidad /retorno sobre el capital de una empresa o

2. Según Irimia (2003), "EVA, es el indicador de creación de valor más popular en la actualidad [...] una medida de beneficio residual que resta el coste de los recursos del beneficio operativo generado en el negocio." (p. 290). negocio sobrepasa sus costos de capital, se está creando valor real para los accionistas.

Utilidad de las actividades ordinarias antes de intereses y después

EVA $=\quad$ de impuestos (UAIDI) menos Valor contable del activo por el costo promedio del capital (Amat, 1999)

EVA $=$ UAIDI $-($ ACTIVOS TOTALES $*$ COSTO DEL CAPITAL)

EVA $=$ NOPAT $-(C O S T O D E$ CAPITAL $*$ ACTIVOS TOTALES $)$

Si el resultado del cálculo del EVA es positivo: se crea valor.

Si el resultado del cálculo del EVA es negativo: se destruye valor.

El UAIDI se obtiene sumando a la utilidad neta los intereses y eliminando las utilidades extraordinarias. En caso de que haya pérdidas extraordinarias, éstas se sumarán a dicha utilidad.

\section{Cálculo del UAIDI}

- Se excluyen las utilidades extraordinarias, para evaluar la operación normal y cotidiana de la empresa.

- Utilidad neta

- $\quad$ - Gastos Financieros

- - Utilidades Extraordinarias

- $\quad$ + Perdidas Extraordinarias

$\cdot \quad=U A|D|$

En el cálculo del EVA se trabaja solamente con utilidades ordinarias porque las extraordinarias podrían desvirtuar la evaluación de la gestión de los responsables de las unidades de negocio y las filiales. Como su denominación lo indica, estas utilidades son atípicas y no están relacionadas con la actividad diaria del negocio. Se considera utilidades extraordinarias las que no están directamente relacionadas con la adminis- 
tración de los responsables. De acuerdo con lo anterior, utilidades extraordinarias pueden ser la compraventa de un activo fijo o pérdidas ocasionadas por fenómenos de la naturaleza, tales como terremotos, huracanes o incendios.

El valor contable del activo es el valor promedio del activo de la empresa, de acuerdo con su balance de situación.

Para el cálculo del activo para el EVA (Guevara et al., 2000), se recomienda

- Usar los valores de mercado de los activos. Habrá que añadir a los valores de adquisición las posibles plusvalías que se hayan generado, en todos los activos fijos o si se dan minusvalías como las depreciaciones se deducen.

- Calcular el valor promedio de los activos utilizados en el periodo.

- Deducir del activo la financiación automática que proporcionan los proveedores, la hacienda pública, seguridad social; para poder trabajar con el activo neto.

Para el análisis del Valor del Negocio, el más popular es el EVA; sin embargo, al ser una marca registrada de Stern Stewart \& Co., dicha compañía y sus respectivas empresas consultoras son las únicas autorizadas para utilizarlo. Esto explica que haya tantas métricas como autores y empresas hay. Según Stern Stewart \& Co. son necesarios hasta 166 ajustes para el EVA, pero muchos estudiosos y otras empresas consultoras dicen que con más o menos 10 ajustes se puede calcular el EVA. Según Bennett Stewart, socio senior de Stern Stewart \& Co. y principal arquitecto de el EVA: "La segunda razón por la que utilizamos valores contables es que hemos descubierto un modo de obviar el problema de los costes históricos: es decir, vinculando las recompensas de la dirección no a las medidas absolutas del EVA, sino a los cambios de un año a otro en el EVA, así como la gestión de la calidad total se centra en la mejora continua de los productos y los procesos, un sistema EVA se centra en la continua mejora de la actuación financiera. Si usted recompensa a la dirección por mejorar el EVA, entonces no tiene realmente importancia el valor que usted asigne a los activos (Rappaport, 2002, p. 89).

Entre los ajustes más frecuentes que afectan el estado de resultados del EVA, se pueden citar:

(Resultado neto $=$ Utilidad neta + ajustes $).$

1. Quitar todos los intereses perdidos.

2. Eliminar todos los intereses ganados.

3. Retirar la amortización de bienes intangibles.

4. Quitar todo costo de I+D, que excedan el $10 \%$ de las ventas del periodo.

5. Eliminar los resultados por actividades no operativas y no vinculadas con el negocio.

6. Retirar resultados extraordinarios.

7. Excluir toda reserva por obsolescencia.

8. Quitar todo cargo de previsión por incobrables.

El objetivo de estos ajustes es la obtención depurada de un resultado neto, obtenido de la actividad económica esencial de la empresa.

Ajuste al costo del capital invertido.

Son el capital de trabajo + Activos no corrientes + ajustes.

Estos tres factores se desglosan de la siguiente manera:

Capital de trabajo: total de activos corrientes - pasivos corrientes, excluyendo los pasivos financieros a corto plazo.

Activos no corrientes: son el total de bienes en uso e intangibles, los cuales se deberán de ajustar. 
Los ajustes son:

- Incorporar los costos de I+D, que excedan el $10 \%$ de las ventas.

- Eliminar del activo, las obras en proceso, acciones, bonos y otros activos que no estén generando utilidades a la hora de la medición.

- Incluir en el activo los valores de los bienes que se encuentren en operaciones de leasing.

- Sumar las provisiones para deudores incobrables y provisiones por obsolescencias.

\section{Costo promedio del capital}

Según Amat (1999): "El costo promedio de capital, es el costo promedio de toda la financiación que ha obtenido la empresa. La empresa necesita obtener financiación para sus inversiones, la cual puede ser interna o externa. Toda financiación, incluido el capital social aportado por los accionistas o la autofinanciación, tiene un costo. Precisamente, uno de los objetivos que debe alcanzar la política financiera de la empresa es que el costo medio de todas las fuentes de financiación sea lo más reducido posible. Este costo medio se denomina costo de capital y se obtiene con base en la medida ponderada del costo de todos los elementos del capital de la empresa" (p. 73).

Un ejemplo práctico en la obtención del EVA se puede apreciar de la siguiente forma:

TABLA 2

GTA, S.A. (NOMBRE FICTICIO)

\begin{tabular}{|c|c|c|c|}
\hline & 2004 & 2005 & 2006 \\
\hline +Utilidad Neta & $\$ 169893$ & $\$ 192662$ & $\$ 214038$ \\
\hline +Gastos Financieros & $\$ 274464$ & $\$ 238872$ & $\$ 218386$ \\
\hline $\begin{array}{l}\text {-Utilidades } \\
\text { Extraordinarias }\end{array}$ & $\$ 26019$ & $\$ 2611$ & $\$ 61258$ \\
\hline $\begin{array}{c}\text { +Pérdidas Extraordi- } \\
\text { narias }\end{array}$ & $\$ 184366$ & $\$ 237171$ & $\$ 269102$ \\
\hline$=$ UAIDI & $\$ 602704$ & $\$ 643094$ & $\$ 640269$ \\
\hline UAIDI & $\$ 602704$ & $\$ 643094$ & $\$ 640269$ \\
\hline Activo * Costo Capital & $\$ 287598$ & $\$ 264268$ & $\$ 190638$ \\
\hline EVA & $\$ 142547$ & $\$ 220266$ & $\$ 335248$ \\
\hline $\begin{array}{c}\text { Incremento Anual, } \\
\text { EVA }\end{array}$ & & $54 \%$ & $52 \%$ \\
\hline
\end{tabular}

FUENTE: Elaboración propia. 
Hoy, los propietarios de la marca de el EVA, han propuesto una "Alternativa para la evaluación de la actuación": el Valor Económico Añadido (VEA).

La segunda versión del beneficio residual, el VEA (Economic Value Added, con su marca registrada EVA) ha sido propuesta por la firma consultora Stern Stewart \& Company. En ella, se ajusta el valor contable a un denominado valor económico contable, añadiendo de nuevo equivalentes al patrimonio neto, tales como las reservas para impuestos diferidos, las reservas por la valoración de existencias LIFO3, la amortización acumulada del fondo de comercio, el fondo de comercio no registrado, las reservas para insolvencias, y las reservas para garantías comprometidas por la empresa. La finalidad de estos ajustes del valor contable es obtener una mejor aproximación de todo el dinero invertido en la empresa" (Rappaport, 2006) ${ }^{4}$.

Como se aprecia al analizar la implantación del Valor del Negocio, muchos de sus tópicos se basan en ajustes contables y financieros, y se echa mano tanto de los ajustes en los activos tangibles, como de los activos intangibles.

Tópicos a tomar en cuenta en el EVA

- La limitante de la UAIDI, es la Contabilidad creativa, es decir, ajustes mal intencionado o maquillaje de la contabilidad para obtener un beneficio.

- De todas las prácticas creativas de la Contabilidad, las relacionadas con el activo fijo y las existencias (inventarios) son las de mayor impacto.

- Recalcular las utilidades introduciendo los ajustes oportunos y pertinentes, para que revelen lo más cerca posible la realidad económica, son recomendaciones de B. Stewart (1998).

3. LIFO: última entrada, primera salida.

4. Para una exposición detallada de este cálculo, ver la obra de G. Bennett Stewart III, The Quest for Value, Nueva York: Harper Collins, 1991, pp 306-350.
- Revisión de los criterios contables para realizar ajustes:

1. Revalorización de activos fijos.

2. Depreciación de activos fijos.

3. Criterios de consolidación.

4. Ajustes por inflación.

5. Conversión de operaciones en divisas.

6. Valoración de inventarios.

7. Gastos de mantenimiento, reparaciones y mejoras.

Con relación al UAIDI:

- Aumentar ventas, para bajar inventarios (activos fijos).

- Reconversión de activos fijos en costos variables para aumentar la flexibilidad en la Empresa.

- Eliminar actividades que no aporten valor a los clientes.

- Reducir activos para directamente reducir gastos relacionados por estos.

- Utilizar servicios de Outsourcing. ${ }^{5}$

- Incrementar la productibilidad en los empleados.

Con el Valor Contable del Activo

Otra forma de aumentar el EVA es reducir el activo o invertir en activos que rindan por encima del costo de capital de la empresa. Algunas formas para reducir el activo:

- Aumentar la rotación de los activos (inventarios), incrementando las ventas.

5. Funciones que no son nucleares para la organización y que pueden ser mejor desempeñadas por otras organizaciones y los beneficios de costos que podrían disminuir por economías de escala o especialización. (Brenes, 2004, p.157). 
- Alquilar activos fijos en vez de adquirirlos.

- Mejorar los procesos, para poder eliminar activos (eliminación de maquinaria).

- Reducir el plazo de inventarios implantando el Justo a Tiempo.

- Implantar técnicas de cobranzas para reducir el plazo de cuentas por cobrar.

- Reducir saldos en Tesorería.

Con relación al Costo Promedio del Capital

- Reducir el costo del capital, a fin de aumentar el valor agregado, variable que incide directamente con el aumento del EVA.

- Si es inevitable el endeudamiento, procurar el costo más bajo de interés, el plazo adecuado y sobre todo invertir solo en negocios rentables.

\section{EVA como método de valuación}

Si bien el EVA es la métrica más popular y es por ello que se le conoce, existe también el Market Value Added (VMA), componente para valuar las empresas, también conocido como Valor de Mercado Agregado.

En el Valor de Mercado Agregado, según Bennett Stewart (1998: p.26), el "EVA depende directamente del valor intrínseco de mercado que tenga dicha empresa". Cuando el Eva es proyectado o descontado a su valor presente, permite ver el valor de mercado creado o el valor de mercado destruido por los responsables de la administración en la empresa.

MVA = Valor de Mercado - Capital, lo que sería lo mismo que el MVA = Valor presente de todos los EVA futuros.

El MVA es la diferencia absoluta entre el valor de mercado de un negocio y su capital. Se diferencia de la tasa de retorno (TIR), que refleja el resultado de un período, en la medida de que el
MVA es una medida acumulativa del desempeño empresarial. Dicha valuación refleja qué tan exitosamente se han ido creando riquezas para el negocio, (Valor del Negocio), así como la inversión de su capital en el pasado y una proyección de cómo sería el negocio si crearan valores económicos; en suma, da una pauta a seguir a los responsables administrativos de la organización. Así las cosas, maximizar el MVA debería ser el objetivo primario de las empresas interesadas en brindar el mayor beneficio económico a sus accionistas.

Para ilustrar la relación entre el EVA y el MVA, se puede recurrir a un ejemplo muy práctico que se da con la relación de un bono en términos de su rendimiento, su presencia en el mercado y su valor en dicho mercado. Si el cupón del bono es mayor que el interés de mercado (costo de capital), el bono podrá ser vendido con prima (posee un EVA positivo, por lo tanto se podrá vender a un MVA positivo). En tanto si el cupón que acompaña al bono está por debajo del interés de mercado, el bono se cotizará a la baja o con descuento. Al ser poseedor de un EVA negativo, se venderá con un MVA negativo.

De esta forma, el logro del objetivo de aumentar y maximizar la creación de ganancias en el Valor del Negocio, redundará en un EVA positivo, lo cual da como resultado el aumento de la creación de Valor del Negocio en el mercado o simplemente un aumento de la prima del valor de la empresa, del MVA.

Se entiende que el MVA es la diferencia entre el valor de mercado total de la empresa (suma del valor de mercado del capital y el valor de mercado de la deuda) y el total de los recursos invertidos para la creación de ese valor (suma de capital aportado por los accionistas y la deuda, aportada por los acreedores) en una fecha -cualquiera que ésta sea-. Como corolario es preciso indicar que si el MVA ha sido positivo, se ha creado valor en el negocio; en consecuencia se ha 
creado valor para el accionista. No así si el MVA es negativo, pues se habría destruido valor para la empresa y por consiguiente para sus accionistas.

A continuación se resume lo anteriormente indicado:

- $\quad M V A=$ Valor de Mercado Total - Capital Total Utilizado

- Valor de Mercado Total = Valor de Mercado del Capital + Valor de Mercado de la Deuda

- $\quad$ Capital Total Utilizado = Patrimonio + Deuda Financiera

Suponiendo que el valor de mercado de la deuda es igual (congruente o simplemente coincide) con su valor contable, se obtiene:

- $\quad$ MVA = Valor de Mercado del Patrimonio - Valor de Mercado de la Deuda

De esta simple expresión, se aprecia y valora la importancia de crear o destruir valor en el negocio, lo cual afecta de forma directa al poseedor de acciones, es decir, a los accionistas del negocio.

Si el resultado del MVA fue positivo o se creó valor, cada colón o unidad de dinero que los accionistas hayan invirtieron en esa empresa, por la buena gestión de sus responsables, hará que obtengan una ganancia; en ese sentido, los accionistas son acreedores directos de la creación de valor, por lo tanto su dinero invertido vale $X$ + \% de creación de valor obtenido por el negocio. En tanto si su MVA es menor o se destruyó valor, cada colón invertido vale $\mathrm{X}$ - \% de destrucción de valor.

\section{Estrategias para aumentar el EVA}

Las estrategias para aumentar el EVA se nombraron en "Tópicos a tomar en cuenta en el EVA." Sin embargo, algunos de los objetivos en la creación de Valor del Negocio, así como ciertas características son de suma importancia para la consecución de estrategias para incrementar el EVA, concepto que, expresado de mejor manera, supone el crear valor en el negocio o empresa.

A continuación se citan algunas estrategias:

- Mejorar e incrementar la eficiencia de los activos que se poseen.

- Bajar o reducir al máximo la carga fiscal.

- Orientar las inversiones en activos que rindan por encima del costo del pasivo.

- Reducir los activos, manteniendo el UAIDI.

- Medidas como la implementación del Justo a Tiempo, orientadas a reducir inventarios, optimizar la producción, producir solo lo que se necesita, etc.

- Alquilar equipos o activos en vez de adquirirlos.

- Reducir el plazo en tiempo y volumen en dinero de las cuentas por cobrar, con el valor esencial de la empresa, el Cliente, razón del porqué y para qué de la empresa o del negocio.

- Hacer más eficiente y eficaz a la Tesorería, reduciendo sus saldos.

- Reducir el costo promedio del Capital, para que así sea menor la deducción que se le hace al UAIDI, por cargo de conceptos a los costos financieros.

El éxito de la puesta en marcha de algunas de estas estrategias, en combinación con otras estrategias no expuestas aquí, permitiría llegar a un aumento del EVA y, por lo tanto crear valor, dando valores reales al negocio y generando dinero, lo que sería el Valor del Negocio.

Es común y muy frecuente que las empresas generen poco EVA, en especial porque al no reducir sus activos, más bien aumentan el valor de sus activos. Esto se da principalmente en sus 
estrategias de incremento de tamaño, que si bien están enfocadas a crear valor en el negocio, implican inversiones que muchas veces no son generadoras de una rentabilidad lo suficientemente alta para crear riqueza y se convierten en destructoras de riquezas.

En consecuencia, el objetivo primo de crear valor, no siempre va de la mano con la empresa, en su objetivo de un mayor tamaño.

Con base en todo lo antes mencionado, y pese a haber sido expuesto de forma bastante general y sintética, se podrían citar cinco estrategias básicas para la incrementar el EVA en una Unidad de Negocio, una Filial o una Empresa, independientemente de que su carácter sea nacional o global:

1. Mejorar la eficiencia de los activos actuales tratando de aumentar el rendimiento de los activos sin invertir más. Para ello, hay que aumentar el margen con el que se vende (a través de un aumento del precio de venta, de reducción de los costos, o con medidas en conjunto); si bien se debe aumentar la rotación de los activos fijos y activos circulantes, para generar más ingresos sin necesidad de aumentar las inversiones. Una de las formas de conseguir aumentar el rendimiento, es invertir en aquellas divisiones que generan valor y no invertir en aquellas unidades productivas que destruyen o generan muy poco valor.

2. Impulsar la reducción de la carga fiscal, implementando una planificación fiscal y tomando decisiones que optimicen las máximas desgravaciones y deducciones fiscales posibles.

3. Aumentar las inversiones en activos que rindan ganancias por encima del costo del pasivo. De esta manera, el incremento de la utilidad superaría el aumento del costo de la financiación, con lo que el EVA sería mayor, se incrementaría.
4. Reducir los activos, manteniendo el UAIDI, para que se pueda disminuir el costo de la financiación total. Es así, como al reducir el pasivo, dicho costo sería menor, siendo esto lo que le resta valor a la utilidad. Por lo tanto, aunque la utilidad (UAIDI) siga siendo la misma, el EVA aumentaría al reducirse el costo financiero, tanto como los activos. Así por ejemplo, se pueden aplicar medidas como el Justo a Tiempo, para bajar los costos del inventario; optar por el alquiler de activos (equipo, maquinaria, entre otras cosas) en vez de comprarlos; reducir el tiempo de las cuentas por cobrar y los saldos en la Tesorería. Este tipo de medidas mantiene el rendimiento con menos inversión.

5. Reducir el costo promedio del capital, para que sea menor la deducción al UAIDI por el concepto de costo financiero. Esta estrategia está muy ligada a la oferta y demanda de los mercados financieros, así como a la confianza que se pueda generar en los acreedores de la empresa, de manera que la consideren de de menor o mayor riesgo, para así poder negociar un costo de capital menos.

Con base en lo expuesto sobre la creación del Valor del Negocio, si se utilizan las estrategias mencionadas, o la combinación de estas estrategias con otras, se puede esperar un aumento en dicho valor, así como en el (EVA) Valor Económico Agregado del negocio -cualquiera que sea el negocio-. Lo anterior, porque al crear valor la empresa, se está creando valor para el accionista, siendo este el fin de toda actividad lucrativa.

A manera de resumen, se citan a continuación algunos de los usos específicos del EVA, en las empresas:

1. Ayuda a fijar metas en la organización.

2. Se utiliza como medición del desempeño.

3. Determinación de bonos (regalías a sus empleados) por desempeño. 
4. Motiva a los gerentes.

5. Visualiza el uso práctico del capital en la empresa.

6. Da una valuación empresarial o corporativa.

7. Sirve para analizar las acciones.

En este siglo XXI, en donde el cambio es la única constante segura, las empresas, hoy más que nunca, se ven motivadas a innovar para poder permanecer en el mundo globalizado de los negocios. En particular, han tenido que cambiar su forma de administrar sus activos tangibles y los activos intangibles.

Así, en los años anteriores y posteriores al 2000, sucede lo mismo. En 1996, por ejemplo, en la bolsa de valores de Japón -la Bolsa de Tokio-, en donde se cotizan las principales empresas japonesas, sucedió que, de la noche a la mañana, los indicadores de la bolsa cambiaron drásticamente; así, el PER (del inglés, Price to Earnings Ratio, o sea la relación entre el precio o valor y los beneficios), que estaba alrededor de 70, descendió a un escalofriante PER de 25, y el índice Nikkei, que rondaba los 40 000, descendió estrepitosamente a 15 000. Literalmente, billones de dólares habían desaparecido. ¿Por qué sucedió esto?

Entretanto, en la acera del frente, la empresa General Electric tenía, para diciembre de 1995, un ROE (Return On Equity, Rentabilidad sobre recursos propios. Mide lo que gana la empresa por cada unidad monetaria invertida en fondos propios) del 23\% y la cotización en la Bolsa de Nueva York era de \$34 / acción; además, mostraba un PER de 17, siendo que el PER promedio de las empresas para esa época era de 19. El CEO de General Electric, Jack Wells, pensó que su compañía estaba infravalorada e inmediatamente pasó a la acción, anunciando la recompra de acciones por 10000 millones de dólares. Fue así como para mediados del año de 1998, la empresa había adquirido acciones por 5000 millones de dólares; para julio de 1998 tenía 3.26 mil de millones de acciones, cotizándose ahora a \$95 / acción, su valor de capitalización era de 310000 millones de dólares en vez de los 113 000 de millones de dólares. Como corolario, con una inversión de 5000 millones de dólares, la empresa había ganado o, mejor dicho, creado valor por casi 200000 millones de dólares y su PER se situaba en 37.

\section{¿Qué sucedió con estos ejemplos cotidianos?}

La buena administración en la toma de decisiones para crear valor y las desacertadas decisiones crearon destrucción de valor. De ahí la enorme importancia de analizar la creación o destrucción de valor en el negocio, para corregir a tiempo los errores y fomentar las decisiones acertadas en este mundo, en donde:

Vemos que los procesos de distribución y los costes se han modificado sustancialmente en una amplia gama de sectores. La desintermediación afecta a la cadena de valor del sector de que se trate, modifica las barreras de entrada, el motor de los beneficios y permite el acceso rápido de nuevos competidores no tradicionales y con espíritu creativo... Esta convergencia de tecnologías y sectores supone la ruptura de fronteras anteriormente muy claramente delimitadas (Parhalad, 2006, p. 90).

El EVA ha cobrado gran importancia, al punto de que, actualmente, alrededor del 29\% de las empresas en los Estados Unidos de América lo están utilizando como método para sacar a flote y crear valor en sus empresas.

\section{CONCLUSIONES}

El crear valor o destruir valor es hoy, más que nunca, un tema de muchísima importancia para 
la sobrevivencia de las empresas; máxime porque muchas veces se invierte más en una empresa que lo que genera para cubrir los costos de oportunidad de su capital de trabajo.

Si la rentabilidad es inferior al costo de oportunidad, cuanto más se invierta en la operación del negocio, más valor se destruye.

Una rentabilidad positiva permite acceder al mercado de capitales, financiar proyectos nuevos e invertir para poder crear más valor en el negocio, siendo el techo de crecimiento de la empresa.

La empresa debe rendir igual que la empresa más exitosa, pues de lo contrario estaría destruyendo valor. En ese sentido, la relación de creación de valor económico y la capacidad de crear conocimiento van de la mano.

Las actividades que según Stern Stewart destruyen valor, son las pre-industriales como: agricultura, minería, ganadería, recursos naturales, manufactura, infraestructura, obras civiles. Lo anterior, debido a que necesitan mucho capital -recursos económicos- y poco capital intelectual.

Por su parte, los negocios que crean valores son: el software, los laboratorios, las marcas, los servicios y las empresas que se basan en el conocimiento para crear valor.

El valor de mercado, por su parte, corresponde al valor de la acción en el futuro y se basa en activos intangibles. Así pues, no solo se mide la relación con los clientes y se valoriza la habilidad competitiva, la creatividad, el conocimiento de los empleados; sino que, además, se miden los procesos internos y la capacidad de crecimiento de la empresa.

El EVA, en su equivalente de capital intelectual, y el MVA, tienen un mayor sustento, para la toma de decisiones en la empresa. El EVA, como se indicó a lo largo de este artículo, es la herramienta financiera para medir la creación de valor y el aporte de los activos intangibles; entre ellos, el capital intelectual, las marcas, las patentes y los derechos de autor, que contribuyen a crear el valor del negocio.

Para determinar la creación de valor en un negocio, es de suma importancia el saber invertiry apoyarse en la métrica del EVA y en el MVA, para constatar la creación de valor, o en su defecto, la destrucción de valor en el negocio.

Las marcas, sin duda alguna, contribuyen de manera importante a dar valor agregado a las empresas exitosas; sin embargo, ninguna empresa es ganadora sin el capital intelectual: los seres humanos son, definitivamente, el motor esencial de toda empresa.

En tanto se cree mayor conciencia respecto al valor que aportan los intangibles a crear valor, en el Valor del Negocio; se irán desarrollando, a su vez, métricas como en el EVA.

\section{REFERENCIAS}

Amat, O. (1999). EVA: valor económico agregado; un nuevo enfoque para optimizar la gestión empresarial, motivar a los empleados y crear valor. Bogotá: Norma.

Arteche, W.; Rozas, W. (1999). Conocimiento Estratégico; crear Valor con la gestión del Conocimiento. Harvard Deusto Business Review.

Brenes, L. (2004). Dirección estratégica para organizaciones inteligentes. San José: EUNED.

Chase, R.; Aguilano, N.; Jacobs, F. (2000). Administración de la producción y Operaciones. México: Ed. Mc Hill.

Collison, C. (2003). La gestión del conocimiento: lecciones prácticas de una empresa líder. Barcelona: Paidós.

Collison, C. (2005). Companies Can Turn Reporting Into Business Value. Quality Progress, 38 (8), 12-20. 
Curley, M. (2006). Managing Information Technology for Business Value. Hillsboro: Intel Press.

Davenport, T. (2006). Motivar, retener y crear valor en la red del conocimiento: conseguir, mejorar el rendimiento y los resultados de los trabajadores del conocimiento. Barcelona: Deusto.

Edvinsson, L.; Malone, M. (1999). El capital intelectual. Madrid: Gestión 2000.

Edvinsson, L.; Malone, M. (2006). Enterprise Resource Planning Systems. Value Implications of Investments in Information Technology. Management Science, 52, 1359-1376.

Faga, H. (2000). Cómo profundizar en el análisis de sus costos para tomar mejores decisiones empresariales. Buenos Aires: Granica.

Garvin, P. (1993). Building a learning organization. Boston: Harvard Business Review

Guevara, T.; Palma, R.; Mena, M. (2000). Análisis para la implementación del enfoque EVA en el Grupo Durman Esquivel. Tesis de maestría en Finanzas.

Harvard Business Review. (1998a). Cómo medir el rendimiento. HDR. Bilbao: Deusto.

Harvard Business Review. (1998b). Gestión de la marca. HDR. Bilbao: Deusto.

Harvard Business Review (1999). On the Business Value of IT. Harvard Business School Press.

Harvard Business Review. (2000). Gestión del conocimiento. HDR. Bilbao: Deusto.

Hay, E. (1989). Justo a tiempo. Bogotá: Ed. Norma.

Henry, D. (1987). Manual de técnicas eficaces para reducir los costos. New Jersey: Alexander Halmiton Institute.

Hidalgo, J. (2006). Generación de valor: en la Economía Global Basada en el Conocimiento. UNED. Propuesta de tesis para optar por el doctorado en Ciencias de la Administración.

Hirchman, A. (1958). The Strategy of Economic Development. New Haven Connecticut: Yale University Press.
Itoh, M.; Urata, S. (1994). Small and medium-size enterprise support policies in Japan. Nr. 1403, Research Working Papers, The Word Bank.

Irimia, A. I. (2003). La creación del valor para el accionista. Madrid: Cia Inversiones Editoriales Dossat 2000.

Janssen, M. (2005). The architecture and business value of semi-cooperative, agent-based supply chain management system. Electronic Commerse Research \& Application, 4, 315-328.

Marchand, D. (2005). Reaping the Business Value of IT. Business \& Economic Review, july-september

Marín, F.; Delgado, J. (2000). Las técnicas de justo a tiempo y su repercusión en los sistemas de producción. Economía Industrial, n. 331-2000/1

Martín, l.; De la Calle, C. (2003). Evolución de la gestión del conocimiento hacia la creación del valor. Universidad Politécnica de Madrid. n.o 17/06-2003

Martín, J. (2001). La gestión basada en el valor: la respuesta de la empresa a la revolución del accionista. Barcelona: Gestión 2000.

Miles, L. (1970). Análisis del valor. Bilbao: Deusto.

Monden, L. (1996). Toyota Production System: an integrated approach to just-in-time., 2 ed. Industrial Engineering and Management.

Nonaka, I.; Ichijo, K.; Van Krogh, G. (2001). Facilitar la creación de conocimiento: Cómo desentrañar el misterio del conocimiento táctico y liberar el poder de la innovación. México: Oxford.

Nonaka, I.; Takeuchi, H. (1995). The Knowledge-Creating Company. Oxford University Press.

Ortiz-Saucedo, S. (2003). Visión y gestión empresarial: cómo articular las estrategias y el capital humano para triunfar en la economía del conocimiento. Madrid:Thonson.

Parlad, C. (2006). Estrategia corporativa. Barcelona: Deusto. 
Parlou, P.; Housel, T.; Rodgers, W.; James, E. (2005). Measuring the Return on Information Technology: A Knowledge-Based Approach for Revenue Allocation at the Process and Firm Level. Journal of the Association for Information Systems, 6 (7), 199-226.

Parro, N. (1972). Análisis del valor; creatividad en la reducción de costos. Buenos Aires: Ed. Contabilidad Moderna.

Peppard, J.; Ward. J. (2005). Unlocking Sustained Business from IT Investment. California Management Review, 48 (1).

Porter, M. (2000). Location, Competition and Economic Development: Local Clusters in a Global Economy. Economic Development Quarterly, 14 (1), 15-34.

Porter, M. (2006a). Estrategia y ventaja competitiva. Barcelona: Deusto.

Porter, M. (2006b). Redefining health care: creating value-based competition on results. Harvard Business School Press.

Rappaport, A. (2006). La creación de valor para el accionista. Barcelona: Deusto.

Reed, R.; Rogers, K.; Johnson, D.; Lies, D. (1996). Engineering the virtual Enterprises. Proceedings of the 5th IERC in Minneapolis, MN.

Sanjurjo, M.; Reinoso, M. (2003). Guía de valoración de empresas. Madrid: Prentice Hall.

Schonberger, R. (1982). Japanese Manufacturing Techniques. New Jersey: The Free Press.
Stern, J. (2002). El reto del EVA: Cómo implantar y gestionar el cambio de valor añadido en una organización. Barcelona: Gestión 2000.

Stewart, B. (1998) Revista-INCAE. Instituto Centroamericano de Administración de Empresas, Volumen 4 - Página 26

Tebo, P. (2005). Building Business Value Through Sustainable Growth Research. Technology Management. 0895-6308/05/S5.00 Septiembre-Octubre.

Thompson, D.; Rust, R.; Rhoda, J. (2005). The business value of e-government for small firms. International Journal of Service Industry Management, 16 (4), 385-407.

Thompson, D.; Rust, R.; Rhoda, J. (2006). Unlocking business value. The conference 2006 Official Report. A supplement to BMJ. August 2006.

Viñolas, P; Adserà, X. (1997). Principios de valoración de empresas. Bilbao: Deusto.

Wareham, J.; Mathiassen, L.; Rai, A.; Klein, R. (2005). The business value of digital supply networks: A program of research on the impacts of globalization. Journal of International Management, 11 (2), 201-227.

Wiig, K. (2000). Knowledge Management: An Emerging Discipline Rooted in long History. Harvard Business Review.

Young, A. (2001). EVA and value-based management: a practical guide to implementation. New York: Mc Graw-Hill.

RECIBIDO: 30-10-2009

ACEPTADO: $13-12-2009$ 